TRANSACTIONS OF THE

AMERICAN MATHEMATICAL SOCIETY

Volume 349, Number 5, May 1997, Pages 2069-2084

S 0002-9947(97)01703-0

\title{
TWIN TREES AND $\lambda_{\Lambda}$-GONS
}

\author{
CURTIS D. BENNETT
}

\begin{abstract}
We define a natural generalization of generalized $n$-gons to the case of $\Lambda$-graphs (where $\Lambda$ is a totally ordered abelian group and $0<\lambda \in \Lambda$ ). We term these objects $\lambda_{\Lambda}$-gons. We then show that twin trees as defined by Ronan and Tits can be viewed as $(1,0)_{\Lambda}$-gons, where $\Lambda=Z \times Z$ is ordered lexicographically. This allows us to then generalize twin trees to the case of $\Lambda$-trees. Finally, we give a free construction of $\lambda_{\Lambda}$-gons in the cases where $\Lambda$ is discrete and has a subgroup of index 2 that does not contain the minimal element of $\Lambda$.
\end{abstract}

\section{INTRODUCTION}

In 1988, M. Ronan and J. Tits [T4] (see [T2] and [T3] also) introduced the notion of twin buildings. These buildings allow one to extend many of the techniques used to study buildings of spherical type to nonspherical buildings. They have also proven useful in the study of Kac-Moody groups and other algebraic groups. As with the case of spherical buildings, twin buildings of rank greater than 3 appear to be highly restricted but very interesting. However, in the rank 2 case, and in particular the case of twin trees, there is much more freedom. Recently M. Ronan and J. Tits [RT] have made great headway in the study of twin trees. They have found many interesting examples and many analogues of results for generalized $n$-gons.

The idea of Ronan and Tits is to pair up two isomorphic trees by use of a codistance function so that there is a similar natural bijection between the neighbors of opposite vertices. Using this property, Ronan and Tits then prove a rigidity theorem for twin trees generalizing the rigidity theorem for generalized $n$-gons. In particular, they show if $\phi$ is an automorphism of a twin tree fixing opposite edges $e_{1}$ and $e_{2}$ and fixing all the neighbors of the vertices of $e_{1}$, then $\phi$ is the identity. Similarly, Ronan and Tits generalize the notion of a Moufang $n$-gon to a twin tree. (For the definition of a Moufang $n$-gon see [R, Chapter 6].) Again, they are able to prove theorems for twin trees that are analogues of theorems for generalized $n$-gons. Throughout their paper, Ronan and Tits use arguments very similar to the arguments used for generalized $n$-gons. While Ronan and Tits use techniques from the study of generalized $n$-gons, there is never any specific link between the two given.

The goal of this paper is to provide a link between twin trees and generalized $n$-gons using $\Lambda$-graphs and $\Lambda$-trees. In section 2 we give the necessary background

Received by the editors April 24, 1994 and, in revised form, January 4, 1996.

1991 Mathematics Subject Classification. Primary 51E12, 20 E99.

Key words and phrases. $\Lambda$-trees, twin trees, generalized $n$-gons.

The author gratefully acknowledges the support of an NSF postdoctoral fellowship. 
material on twin trees and $\Lambda$-trees. In section 3 we introduce the notion of a $\Lambda$-graph and prove that any $\Lambda$-graph is covered by a $\Lambda$-tree. We note that this theorem is, in fact, a special case of the work of Basarab ([B]). However, it is much easier to give a direct proof of the covering theorem, as we do in this paper, than to prove that our $\Lambda$-graphs satisfy Basarab's conditions. Section 4 of the paper defines $\lambda_{\Lambda}$-gons and shows that for $\Lambda=Z$ these are just generalized $n$-gons. We then show:

Theorem 1.1. Let $\Lambda=Z \times Z$ be lexicographically ordered. If $\mathcal{T}$ is a twin tree, then $\Phi_{n}(\mathcal{T})$ is a generalized $(1, n)_{\Lambda}$-gon for all $n \in Z$.

This theorem also gives us a natural way to generalize the definition of a twin tree to the case of $\Lambda$-trees. Essentially, we define a twin $\Lambda$-tree as a $Z \times \Lambda$-graph satisfying

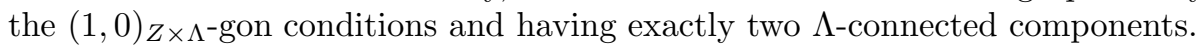

Using the covering theorem for $\Lambda$-graphs, we next obtain:

Theorem 1.2. Let $\Lambda=Z \times Z$. Given a twin tree $\mathcal{T}$, there exist a $\Lambda$-tree $\mathcal{C}(\mathcal{T})$ and a group $G$ of deck transformations of $\mathcal{C}(\mathcal{T})$ such that $\mathcal{C}(\mathcal{T})$ is a universal cover of $\mathcal{T}$, by which we mean that $\mathcal{C}(\mathcal{T})$ covers the associated $(1,0)_{\Lambda}-$ gon $\Phi_{0}(\mathcal{T})$ and

$$
\Phi_{0}(\mathcal{T}) \cong \mathcal{C}(\mathcal{T}) / G .
$$

Section 5 presents a free construction of generalized $\lambda_{\Lambda}$-gons generalizing both the standard free construction for generalized $n$-gons and the free construction of twin trees given in $[\mathrm{Be}]$.

In a future paper, we shall attempt to generalize many of the theorems of Ronan and Tits to the case of $\lambda_{\Lambda}$-gons. At this point, we would like to thank M. Ronan and J. Tits for providing us with a preprint of their paper, and for many helpful discussions on these and related topics. We would also like to thank P. Shalen for directing us to known work on the subject of $\Lambda$-graphs.

\section{Preliminaries}

We will be using ideas from three main areas in this paper: the study of twin trees, the study of $\Lambda$-trees and $\Lambda$-graphs (where $\Lambda$ is a totally ordered abelian group), and the notion of a generalized $n$-gon.

Twin Trees and $n$-gons. Throughout this section we will follow much of the work of M. Ronan and J. Tits [RT].

Definition. A twin tree is a triple $\left(T_{+}, T_{-}, \hat{d}\right)$ such that $T_{+}$and $T_{-}$are (combinatorial) trees, and

$$
\hat{d}:\left(T_{+} \times T_{-}\right) \cup\left(T_{-} \times T_{+}\right) \rightarrow Z_{\geq 0}
$$

is a symmetric function satisfying the following conditions:

1. $\hat{d}(x, y) \geq 0$ for all $(x, y)$ in the domain of $\hat{d}$.

2. If $\hat{d}(x, y)=m$, and $y \sim y^{\prime}$ (where $y \sim y^{\prime}$ means that $\left(y, y^{\prime}\right)$ is an edge of one of the trees), then $\hat{d}\left(x, y^{\prime}\right)=m \pm 1$.

3. If $\hat{d}(x, y)=m>0$, then there is a unique $y^{\prime}$ adjacent to $y$ (in $T_{+}$or $T_{-}$as $y$ is) with $\hat{d}\left(x, y^{\prime}\right)=m+1$.

We term $\hat{d}(x, y)$ the codistance of $x$ and $y$. We call the twin tree $\mathcal{T}$ thick if all vertices of $T_{+}$and all the vertices of $T_{-}$have valency at least 3 . Define vertices $x \in T_{+}$and $y \in T_{-}$to be opposite if $\hat{d}(x, y)=0$. 
Following [RT], given a tree in which every vertex has valency at least 2 , an apartment is a path having no end points. A half-apartment is a path with only one end point. Two half-apartments have the same end if their intersection is a half-apartment. This defines a natural equivalence relation on the set of halfapartments, and we call these equivalence classes the ends of the tree.

It is now an easy consequence of the definition of a twin tree that two nonopposite vertices determine unique ends of $T_{+}$and $T_{-}$by taking in $T_{+}$the path $\left(x_{0}, x_{1}, \ldots\right)$ of vertices starting with $x_{0}=x$ and letting $x_{i}$ be the unique vertex adjacent to $x_{i-1}$ whose codistance from $y$ is larger. Similarly we define an end of $T_{-}$.

As a consequence of this definition, Ronan and Tits [RT, Proposition 3.4] prove:

Proposition 2.1. Suppose the ends $e_{+}$and $e_{-}$are determined by vertices $x$ and $y$ of the twin tree $\mathcal{T}$, and the ends $e_{+}^{\prime}, e_{-}^{\prime}$ are determined by the vertices $x^{\prime}$ and $y^{\prime}$ of $\mathcal{T}$. Then $e_{+}=e_{+}^{\prime}$ if and only if $e_{-}=e_{-}^{\prime}$. Given $x_{0} \in T_{+}$and $y_{0} \in T_{-}$with $\hat{d}\left(x_{0}, y_{0}\right)=m>0$, there exist unique sequences $\left(x_{0}, x_{1}, \ldots\right)$ and $\left(y_{0}, y_{1}, \ldots\right)$ such that $\hat{d}\left(x_{i}, y_{j}\right)=m+i+j$.

Using the standard definitions of graph theory, for $n \in Z_{+}$, we define a generalized $n$-gon to be a (combinatorial) bipartite graph $G$ with diameter $n$ and girth $2 n$.

We state the following standard result on generalized $n$-gons (see $[\mathrm{K}]$ ).

Proposition 2.2. Let $G$ be a generalized n-gon, and $x, y \in G$ arbitrary vertices. Then

1. If $d(x, y)<n$ there exists a unique shortest path from $x$ to $y$.

2. If $d(x, y)=n$, then $x$ and $y$ have the same valency (that is, the same number of neighbors in $G$ ).

3. Every path in $G$ of length $n+1$ is contained in a unique circuit of length $2 n$.

$\Lambda$-trees. Let $\Lambda$ be a totally ordered abelian group. For $\lambda_{1}, \lambda_{2} \in \Lambda$, define $\left[\lambda_{1}, \lambda_{2}\right]=$ $\left\{\alpha \in \Lambda \mid \lambda_{1} \leq \alpha \leq \lambda_{2}\right\}$. We similarly define the open and half open intervals of $\Lambda$.

Given a set $T$, a $\Lambda$-metric on $T$ is a function $d: S \times S \rightarrow \Lambda_{\geq 0}$ such that for all $x, y, z \in T$ we have

(i) $d(x, y)=d(y, x)$;

(ii) $d(x, y) \geq 0$ with equality if and only if $x=y$; and

(iii) $d(x, y) \leq d(x, z)+d(y, z)$.

In this case we call the pair $(T, d)$ a $\Lambda$-metric space. We note that $(\Lambda, d)$ is a $\Lambda$ metric space when $d: \Lambda \times \Lambda \rightarrow \Lambda$ is defined by $d\left(\lambda_{1}, \lambda_{2}\right)=\left|\lambda_{1}-\lambda_{2}\right|$. Let $\left(S, d_{1}\right)$ and $\left(T, d_{2}\right)$ be two $\Lambda$-metric spaces; a $\Lambda$-isometry is an injective map $f: S \rightarrow T$ such that for all $x, y \in S$, we have $d(x, y)=d(f(x), f(y))$.

Definition. The pair $(T, d)$ is a $\Lambda$-tree if $T$ is a set and $d$ is a $\Lambda$-metric on $T$ such that the following conditions are satisfied:

1. For all $x, y \in T$, there exists a unique $\Lambda$-isometry

$$
f_{x, y}:[0, d(x, y)] \rightarrow T
$$

such that $f_{x, y}(0)=x$ and $f_{x, y}(d(x, y))=y$. Define $[x, y]=\operatorname{Image}\left(f_{x, y}\right)$.

2. (Y-condition) Given $x, y, z \in T$, there exists $w \in T$ such that

$$
[x, y] \cap[x, z]=[x, w] ;
$$

3. (Acyclic condition) If $[x, y] \cap[y, z]=\{y\}$ then $[x, z]=[x, y] \cup[y, z]$. 
Let $T$ be a $\Lambda$-tree. Given $x \in T$, an open $x$-ray of $T$ is an infinite (possibly uncountable) union $\bigcup_{i}\left[x, y_{i}\right]$ where $\left[x, y_{i}\right] \subset\left[x, y_{j}\right]$ whenever $i<j$, and the containment is proper. Maximal open $x$-rays of $T$ are called open $T$-rays from $x$. Let $R$ and $R^{\prime}$ be two open $T$-rays (from possibly different points). We define $R$ and $R^{\prime}$ to be equivalent if $R \cap R^{\prime}$ is an open T-ray. This is an equivalence relation whose classes are called the ends of $T$. Alperin and Bass [AB, (2.24)] show that, given an end $\epsilon$ and vertex $x$ of $T$, there is a unique open $T$-ray based at $x$ with end $\epsilon$. Let this ray be denoted $[x, \epsilon)$.

Definition. The triple $(T, d, v)$ is a rooted $\Lambda$-tree if $(T, d)$ is a $\Lambda$-tree, and $v$ is either a vertex or an end of $T$. We say that $v$ is the root of $(T, d, v)$. This definition varies slightly from that of $[\mathrm{AB}]$, as they only allow for a $\Lambda$-tree to be rooted at a vertex.

We will use an equivalent definition of a rooted $\Lambda$-tree with a semi-lattice flavor. Given a set $T$ and a partial order $\leq$ on $T$, we say that $(T, \leq)$ is a tree if for all $x \in T$, the set $\{y \in T \mid x \leq y\}$ is totally ordered under $\leq$. The pair $(T, \leq)$ is an upper semi-lattice, if every pair of elements $a, b \in T$ has a least upper bound $a \vee b$ in $T$. We now state without proof an elementary but useful proposition.

Proposition 2.3. Given an upper semi-lattice tree $(T, \leq)$ and points $a, b, c \in T$, we have that at least two of the elements $a \vee b, a \vee c$, and $b \vee c$ are equal.

Given a rooted $\Lambda$-tree $(T, d, v)$, we define a partial order $\leq$ on $T$ by letting $x \leq y$ if and only if $[y, v] \subseteq[x, v]$ if $v$ is a vertex, and $x \leq y$ if and only if $[y, \epsilon) \subseteq[x, \epsilon)$ if $v=\epsilon$ is an end. In this case, $(T, \leq)$ is a upper semi-lattice tree. In the case where $v$ is a vertex, this follows as the Y-condition implies that for all $x, y \in T$, we have $[x, v] \cap[y, v]=[w, v]$ for some $w \in T$. Similarly the Y-condition implies the result for $v$ an end of $T$. Since for all $x \in T$, the set $\{y \in T \mid x \leq y\}=[x, v]$ for $v$ a vertex (similarly $[x, \epsilon)$ for $v$ an end), we see that $(T, \leq)$ is an upper semi-lattice tree.

Definition. A semi-lattice $\Lambda$-tree is a triple $(T, \leq, \delta)$ such that $(T, \leq)$ is an upper semi-lattice tree, and

$$
\delta:\{(x, y) \in T \times T \mid x \leq y \text { or } y \leq x\} \rightarrow \Lambda_{\geq 0},
$$

such that for all $x, y \in T$ with $x \leq y$ there exists a $\Lambda$-isometry

$$
f_{x, y}:[0, \delta(x, y)] \rightarrow\{z \in T \mid x \leq z \leq y\}
$$

with $f_{x, y}(0)=x$ and $f_{x, y}(\delta(x, y))=y$. We define the set $[x, y]$ to be the image of $f_{x, y}$, which is of course $\{z \in T \mid x \leq z \leq y\}$.

Clearly from the above we have that a rooted $\Lambda$-tree uniquely determines a semilattice $\Lambda$-tree in the fashion stated above. Interestingly, there is an inverse process taking a semi-lattice $\Lambda$-tree to a rooted $\Lambda$-tree.

Given a semi-lattice $\Lambda$-tree $(T, \leq, \delta)$, define $d: T \times T \rightarrow \Lambda$ by $d(a, b)=\delta(a, a \vee b)$ $+\delta(b, a \vee b)$. It is immediate that $d$ is a symmetric function on $a$ and $b$. Suppose $d(a, b)=0$; then $\delta(a, a \vee b)=0$, the isometry condition on $T$ forces $a=a \vee b=b$. Given $x, y, z \in T$, an easy check using Proposition 2.3 shows that $d(x, z) \leq d(y, z)+$ $d(x, y)$ and thus $d$ satisfies the triangle inequality. We claim that $(T, d)$ is a $\Lambda$-tree. To check this we need to check the three conditions on $(T, d)$ in the definition of a $\Lambda$-tree. The existence of an isometry from $[0, d(x, y)] \rightarrow T$ is easily verified by pasting together the isometries from $[0, \delta(x, x \vee y)]$ and $[0, \delta(y, x \vee y)]$ to $[x, x \vee y]$ 
and $[y, x \vee y]$ respectively. The uniqueness condition follows since $(T, \leq)$ is an upper semi-lattice tree, and any element $c$ with $d(x, c)+d(y, c)=d(x, y)$ must lie in either $[x, x \vee y]$ or $[y, x \vee y]$. Now, for $x, y \in T$, we have that $[x, y]=[x, x \vee y] \cup[x \vee y, y]$. The Y-condition follows from a simple case by case analysis using Proposition 2.3. The acyclic condition also follows from Proposition 2.3. If $T$ has a maximal element, let $v$ be the maximal element, otherwise let $v$ be the end associated to the open $x$-ray $\{y \in T \mid x \leq y\}$. Then, $(T, d, v)$ is a rooted $\Lambda$-tree. Moreover we have shown:

Proposition 2.4. A rooted $\Lambda$-tree $(T, d, v)$ uniquely determines a semi-lattice $\Lambda$ tree $(T, \leq, \delta)$, where $\leq$ is determined by the root $v$, and $\delta$ is the restriction of $d$ to the set $\Omega=\{(x, y) \in T \times T \mid x \leq y$ or $y \leq x\}$. Similarly, a semi-lattice $\Lambda$-tree uniquely determines a rooted $\Lambda$-tree by the process given in the preceding paragraph. Moreover, these two processes are inverse.

\section{3. $\Lambda$-Distance Graphs}

Before we define a generalized $\lambda_{\Lambda}$-gon we need a general definition of a $\Lambda$-distance graph.

Definition. Let $\mathcal{G}=(\mathcal{P}, d)$, where $\mathcal{P}$ is a set and $d$ is a $\Lambda$-metric on $\mathcal{P}$. Let

$$
\mathcal{F}_{\mathcal{G}}=\left\{f:\left[0, \lambda_{f}\right] \rightarrow \mathcal{P} \mid \lambda_{f} \in \Lambda \text {, and } f \text { a } \Lambda \text {-isometry }\right\} .
$$

We say that $\mathcal{G}$ is a $\Lambda$-distance graph if the following conditions are satisfied:

1. For all $x, y \in \mathcal{P}$, there exists $f \in \mathcal{F}_{\mathcal{G}}$ such that $\lambda_{f}=d(x, y), f(0)=x$, and $f(d(x, y))=y$.

2. For all $f, g \in \mathcal{F}_{\mathcal{G}}$ with $f\left(\lambda_{f}\right)=g(0)$, there exists $a_{f g} \in\left[0, \lambda_{f}\right]$ with $a_{f g} \leq \lambda_{g}$ such that

(i) $g(x)=f\left(\lambda_{f}-x\right)$ for all $x \in\left[0, a_{f g}\right]$; and

(ii) (Y-Condition) If $b \in \Lambda$ with $b>a_{f g}$, then there exists $x \in\left[a_{f g}, b\right]$ such that $g(x) \neq f\left(\lambda_{f}-x\right)$.

In [B], Basarab defines a more general $\Lambda$-graph which allows for multiple edges and loops in $Z$-graphs. This definition is quite complicated, however, and while the above is a special case, this is extremely difficult to show.

Note. As an easy consequence of the definition, we note that for $f \in \mathcal{F}_{\mathcal{G}}$, the function $f^{-1}(x)=f\left(\lambda_{f}-x\right)$ is a $\Lambda$-isometry, and for $f, g$ as in 2, we have $a_{f g}=$ $a_{g^{-1} f^{-1}}$.

It is straightforward to see that $\Lambda$-trees, combinatorial graphs, and the unit circle in $R^{2}$ are all $\Lambda$-graphs with $\Lambda=Z$ in the second case, and $\Lambda=R$ in the third.

Definition. Let $\mathcal{G}=(\mathcal{P}, d)$ be a $\Lambda$-graph. For $f, g \in \mathcal{F}$ with $f\left(\lambda_{f}\right)=g(0)$, we define the non-backtracking concatenation $f * g$ of $f$ and $g$ by

$$
(f * g)(x)= \begin{cases}f(x) & \text { for } x \in\left[0, \lambda_{f}-a_{f g}\right], \\ g\left(x-\lambda_{f}+2 a_{f g}\right) & \text { for } x \in\left[\lambda_{f}-a_{f g}, \lambda_{g}+\lambda_{f}-2 a_{f g}\right],\end{cases}
$$

We then define the length of $f * g$ to be $l(f * g)=\lambda_{f}+\lambda_{g}-2 a$. It is immediate that $(f * g)^{-1}=g^{-1} * f^{-1}$. The non-backtracking concatenation $f * g$ can be thought of as the path obtained by following $f$ and then $g$, but eliminating the portion over which you backtracked.

Proposition 3.1. Let $\mathcal{G}=(\mathcal{P}, d)$ be a $\Lambda$-graph and $\mathcal{F}$ the associated set of isometries from intervals of $\Lambda$ to $\mathcal{G}$. Suppose $f, g, h \in \mathcal{F}$ are such that $f\left(\lambda_{f}\right)=g(0)$ and 
$g\left(\lambda_{g}\right)=h(0)$. Then $f *(g * h)=(f * g) * h$, and hence the operation $*$ is associative whenever it makes sense.

Proof. The proof is tedious but straightforward. We will leave the details to the reader with the following comments. If $a_{f g}+a_{g h}<\lambda_{g}$, one simply writes down the two functions and sees that they are equal since $a_{(f g) h}=a_{g h}$ and $a_{f(g h)}=a_{f g}$. If $a_{f g}+a_{g h} \geq \lambda_{g}$, then the proof becomes a little harder. Writing down the functions, the result rests on showing that $f\left(x+\lambda_{f}-a_{f g}\right)=h\left(x+\lambda_{g}-a_{f g}\right)$ for $x \in\left[0, a_{g h}+a_{f g}-\lambda_{g}\right]$, which follows from the definitions of $a_{f g}, a_{g h}$, etc.

Definition. In general, we define a path of $\mathcal{G}$ to be $P=f_{1} * \cdots * f_{k}$, with the obvious requirements on the $f_{i}$, and given a path $P$, inductively define the length of $P$ to be

$$
l(P)=l\left(\left(f_{1} * \cdots * f_{k-1}\right) * f_{k}\right) .
$$

For convenience, we will often write $\lambda_{P}$ in place of $l(P)$. It is immediate that $l(P)=\sum\left(l\left(f_{i}\right)\right)-2 \alpha$ for some $\alpha \in \Lambda$. Note that given a path $P$, by the $\mathrm{Y}$-condition there exist $x_{0}, x_{1}, \ldots, x_{k}=x_{0}$, such that $l(P)=d\left(x_{0}, x_{1}\right)+\cdots+d\left(x_{k-1}, x_{k}\right)$.

Definition. Let $\mathcal{G}=(\mathcal{P}, d)$ be a $\Lambda$-distance graph. We define the diameter of $\mathcal{G}$ to be

$$
\operatorname{diam}(\mathcal{G})=\sup _{x, y \in \mathcal{P}}\{d(x, y)\}
$$

We say that $\mathcal{G}$ achieves its diameter if there exist $x, y \in \mathcal{P}$ such that $d(x, y)=$ $\operatorname{diam}(\mathcal{G})$. Note that it is possible both for the diameter of $\mathcal{G}$ to be undefined and for $d(x, y)$ to have an upper bound.

Definition. Let $\mathcal{G}=(\mathcal{P}, d)$ be a $\Lambda$-distance graph. A circuit of $\mathcal{G}$ is a path $P=$ $f_{1} * \cdots * f_{k}$ such that $\lambda_{P}>0$ and $P(0)=P\left(\lambda_{P}\right)$. Define the girth of $\mathcal{G}$ to be the $\inf _{P}\left(\lambda_{P}\right)$, where the infimum is taken over the set of all circuits of $G$. We say that $\mathcal{G}$ achieves its girth, if there exists a circuit $P$ of $G$ such that $\lambda_{P}$ is the girth of $\mathcal{G}$. Again, it is possible that the girth of $\mathcal{G}$ could be undefined in $\Lambda$. If $\Lambda$ is discrete, then any $\Lambda$-distance graph having a diameter and girth in $\Lambda$, achieves both.

We close this section with the covering theorem of Basarab.

Theorem 3.2. Let $\Lambda$ be a totally ordered abelian group. Given a $\Lambda$-distance graph $\mathcal{G}=(\mathcal{P}, d)$, then there exist a $\Lambda$-tree $\mathcal{T}=(T, \tilde{d})$ and a group $H$ acting on $(T, \tilde{d})$ without fixed points such that $\mathcal{G} \cong \mathcal{T} / H$.

Proof. Choose a base point $x \in \mathcal{P}$. Let $\left(S, \leq_{x}\right)$ be the partially ordered set of all paths of $\mathcal{G}$ with initial vertex $x$ where the order is inclusion. We claim that $\left(S, \leq_{x}\right)$ is an upper semi-lattice tree. That $\left(S, \leq_{x}\right)$ is an upper semi-lattice follows from the $\mathrm{Y}$-condition for $\Lambda$-distance graphs. That it is a tree, is immediate since the set of subpaths based at $x$ of a path $P$ is linearly ordered by inclusion. Define $d: \Omega \rightarrow \Lambda$ by $d\left(P_{1}, P_{2}\right)=l\left(P_{2}^{-1} * P_{1}\right)$. As $d\left(P_{1},-\right)$ is an isometry from the subpaths of $P_{1}$ based at $x$ to $\left[0, l\left(P_{1}\right)\right]$, it follows that $\left(S, \leq_{x}, d\right)$ is a lattice $\Lambda$-tree. Let $\mathcal{T}=(T, \tilde{d})$ be the underlying $\Lambda$-tree.

Now suppose $C$ is a circuit of $\mathcal{G}$ based at $x$. The bijection $\phi_{C}: S \rightarrow S$ defined by

$$
\phi_{C}(P)=C * P
$$


acts as an isometry on $\mathcal{T}$. Let $H=\left\langle\phi_{C}\right| C$ a circuit of $\left.\mathcal{G}\right\rangle$. It is immediate that $H$ is a group of isometries of $\mathcal{T}$. Moreover, no element of $H$ can fix any element of $S$. Define $\mathcal{T} / H=\left(\mathcal{P}^{\prime}, d^{\prime}\right)$ by letting $\mathcal{P}$ be the set of equivalence classes of points of $T$ under $H$, and letting $d^{\prime}\left(\overline{P_{1}}, \overline{P_{2}}\right)$ be the minimal distance between any two representatives of $\overline{P_{1}}$ and $\overline{P_{2}}$. Such a minimum exists since the representatives of $\overline{P_{i}}$ are just the paths whose terminal vertex is the terminal vertex $v_{i}$ of $P_{i}$, and there is a minimal path $P$ between $v_{1}$ and $v_{2}$ in $\mathcal{G}$. Thus the path in $\mathcal{T}$ which is the lift of $P$ is a path of minimal length from $\overline{P_{1}}$ to $\overline{P_{2}}$. We now claim that $\mathcal{G} \cong \mathcal{T} / H$. Let $\Psi: \mathcal{T} / H \rightarrow \mathcal{G}$ be defined by $\Psi(P)=P(l(P))$, i.e. the terminal vertex of $P$. It is now an easy check to see that $d^{\prime}\left(\overline{P_{1}}, \overline{P_{2}}\right)=d\left(\Psi\left(P_{1}\right), \Psi\left(P_{2}\right)\right)$, since $\Psi$ maps paths in $\mathcal{T}$ to paths in $\mathcal{G}$.

\section{4. $\Lambda$-GONS}

We now wish to study a special case of $\Lambda$-distance graphs generalizing the notion of generalized $n$-gons. Unfortunately, the most obvious way of generalizing the definition fails to satisfy certain of the natural conditions we wish to have.

For $P$ a path of a $\Lambda$-distance graph $\mathcal{G}$, and $\lambda<\lambda_{P}$, we define $\left.P\right|_{\lambda}$ to be the path restricted to the interval $[0, \lambda]$ of its domain.

Definition. Let $\Lambda$ be a totally ordered abelian group, and $\lambda \in \Lambda$ with $\lambda>0$. A generalized $\lambda_{\Lambda}$-gon is a $\Lambda$-distance graph $\mathcal{G}$ of diameter $\lambda$ and girth $2 \lambda$ such that for all paths $P$ of $\mathcal{G}$ with $\lambda_{P}>\lambda$ there exist a $\alpha \in\left(\lambda, \lambda_{P}\right]$ and a circuit $C$ of $\mathcal{G}$ such that $\left.P\right|_{\alpha} \subset C$ and $\lambda_{C}=2 \lambda$.

Notation. We will simply write $\lambda$-gon for $\lambda_{\Lambda}$-gon if the context allows.

Proposition 4.1. Let $\mathcal{G}$ be $a \lambda_{\Lambda}$-gon. Let $x, y$ be points of $\mathcal{G}$ with $d(x, y)<\lambda$. Then there exists a unique path from $x$ to $y$ of length less than $2 \lambda-d(x, y)$. Moreover, $\mathcal{G}$ achieves both its girth and its diameter.

Proof. The first claim follows since $\lambda_{f * g}>0$ if $f \neq g$, and if there were paths $P_{1}$ and $P_{2}$ from $x$ to $y$ with $P_{1} \neq P_{2}$, then $C=P_{1} *\left(P_{2}\right)^{-1}$ would be a circuit of length less than $2 \lambda$. The second claim follows from the definition.

Proposition 2.2 implies that generalized $n$-gons with $n \in Z^{+}$are in fact generalized $n_{Z}$-gons. The following proposition shows that all generalized $n_{Z}$-gons are generalized $n$-gons.

Proposition 4.2. Let $\mathcal{G}=(\mathcal{P}, d, \mathcal{F})$ be a generalized $n_{Z}-$ gon. Then $\mathcal{G}$ is a generalized n-gon, where $\mathcal{P}$ is the set of vertices of $\mathcal{G}$, and $\{x, y\}$ is an edge of $\mathcal{G}$ if $d(x, y)=1$.

Proof. It suffices to show that $\mathcal{G}$ is a bipartite graph, since the girth of $\mathcal{G}$ is $2 n$ and the diameter of $\mathcal{G}$ is $n$ by the definition of $\mathcal{G}$. Now, suppose

$$
C=x_{0}, x_{1}, \ldots, x_{l}=x_{0}
$$

is a circuit of minimal odd length greater than $2 n$. Then, since $P=x_{0}, \ldots, x_{n}$ is a path of length $n+1$, there exists a circuit of length $2 n$ in $\mathcal{G}$ containing $P$. This implies there is a path from $x$ to $x_{n}$ of length $n-1$. Thus we obtain a circuit $C^{\prime}$ by beginning on this path of length $n-1$. However, the length of $C^{\prime}$ is 2 less than the length of $C$. But $C^{\prime}$ also has odd length greater than $2 n$, contradicting the choice of $C$. 
When $\Lambda$ is discrete we can simplify the definition of a $\lambda$-gon slightly so that it closely resembles the definition of an $n$-gon in the usual sense.

Proposition 4.3. Let $\Lambda$ be a discrete totally ordered abelian group with minimal positive element 1 . Then, $\mathcal{G}$ is a generalized $\lambda$-gon if and only if $\mathcal{G}$ is a $\Lambda$-distance graph of diameter $\lambda$ and girth $2 \lambda$ having no circuits of length $2 \lambda+1$.

Proof. Let $\mathcal{G}$ be a generalized $\lambda$-gon. We need only see that $\mathcal{G}$ has no circuits of length $2 \lambda+1$. Suppose $C=f_{1} * \cdots * f_{k}$ is a circuit of length $2 \lambda+1$. Then by Proposition 4.1 there exist points $a, b, c$ in $C$ such that $d(a, b)=d(a, c)=\lambda$, and $d(b, c)=1$. Letting $P$ be the path following $C$ from $a$ to $b$ and continuing to $c$, from the definition of a generalized $\lambda$-gon we have that there exists a circuit of length $2 \lambda$ containing $P$, since 1 is a minimal positive element of $\Lambda$. However, this implies $d(a, c)<\lambda$, a contradiction.

For the reverse direction, we simply need to see that $\mathcal{G}$ satisfies the property that any path $P$ of length greater than $\lambda$ has a restriction of length greater than $\lambda$ contained in a circuit of length $2 \lambda$. Consider $\left.P\right|_{\lambda+1}$. Let $a$ be the initial point of $P, b$ the point of $\left.P\right|_{\lambda+1}$ at distance $\lambda$ from $a$, and $c$ be the terminal point of $\left.P\right|_{\lambda+1}$. Then since $d(a, c) \leq \lambda$, there exists a path $P^{\prime}$ from $a$ to $c$ of distance less than or equal to $\lambda$. However, $\lambda_{P^{\prime}}<\lambda$, since otherwise $P *\left(P^{\prime}\right)^{-1}$ would be a circuit of length $2 \lambda+1$. Thus $P *\left(P^{\prime}\right)^{-1}$ must be a circuit of length $2 \lambda$.

Suppose $\Lambda$ is a discrete totally ordered abelian group and $\mathcal{G}$ is a generalized $\lambda$ gon. Let 1 be the minimal positive element of $\Lambda$ and define $x, y \in \mathcal{G}$ to be adjacent if $d(x, y)=1$.

The following is an immediate corollary of the preceding proposition.

Corollary 4.4. Let $\Lambda$ be a discrete totally ordered abelian group with minimal element $1, \lambda \in \Lambda$, and $\mathcal{G}$ a $\Lambda$-distance graph of diameter $\lambda$ and girth $2 \lambda$. Then $\mathcal{G}$ is a generalized $\lambda$-gon if and only if whenever $x, y$ are adjacent in $\mathcal{G}$, then for all $w \in \mathcal{G}$ we have $d(w, x)=d(w, y) \pm 1$.

We are now ready to prove one of our main theorems relating $\lambda_{\Lambda}$-gons and twin trees.

Let $\mathcal{T}=\left(T_{+}, T_{-}, \hat{d}\right)$ be a twin tree. Let $\Lambda=Z \times Z$. Let $\mathcal{P}=T_{+} \cup T_{-}$, and let $d_{n}: \mathcal{P} \times \mathcal{P} \rightarrow \Lambda$ be defined as follows:

$$
d_{n}(x, y)= \begin{cases}\left(0, d_{+}(x, y)\right), & x, y \in T_{+}, \\ \left(0, d_{-}(x, y)\right), & x, y \in T_{-}, \\ (1, n-\hat{d}(x, y)), & \text { otherwise }\end{cases}
$$

Define $\Phi_{n}(\mathcal{T})=\left(\mathcal{P}, d_{n}\right)$.

Theorem 4.5. Let $\mathcal{T}$ be a twin tree and $\Lambda=Z \times Z$, ordered lexicographically. Then $\Phi_{n}(\mathcal{T})$ is a generalized $(1,0)$-gon.

Proof. We first show that $\Phi_{n}(\mathcal{T})$ is a $\Lambda$-distance graph. The function $d_{n}$ is symmetric since $\hat{d}$ is symmetric. Similarly, condition 2 of the definition of a twin tree implies that $d_{n}$ satisfies the triangle inequality. Hence $d_{n}$ is a $\Lambda$-metric.

Condition 1 in the definition of a $\Lambda$-distance graph is immediate if $x$ and $y$ lie in the same tree. Suppose $x \in T_{+}$and $y \in T_{-}$. By Proposition 2.1, there exist sequences $\left(x_{0}, x_{1}, \ldots\right)$ and $\left(y_{0}, y_{1}, \ldots\right)$ with $x=x_{0}$ and $y=y_{0}$ such that $\hat{d}\left(x_{i}, y_{j}\right)=\hat{d}(x, y)+i+j$ and $x_{i}$ is adjacent to $x_{i-1}$ and $y_{i}$ is adjacent to $y_{i-1}$ for 
all $i$. Moreover, these sequences are unique if $\hat{d}(x, y)>0$. Let $\hat{d}(x, y)=m$, and define $f_{x, y}:[(0,0),(1, n-m)] \rightarrow T$ by

$$
f_{x, y}(s, t)= \begin{cases}x_{t} & \text { if } s=0 \\ y_{n-m-t^{\prime}} & \text { if } s=1 .\end{cases}
$$

We then have

$$
\begin{aligned}
d_{n}\left(x_{t}, x_{t}^{\prime}\right) & =\left(0,\left|t-t^{\prime}\right|\right), \\
d_{n}\left(y_{m-t}, y_{m-t^{\prime}}\right) & =\left(0,\left|t-t^{\prime}\right|\right), \text { and } \\
d_{n}\left(x_{t}, y_{m-t^{\prime}}\right) & =\left(1, n-m+t^{\prime}-t\right) .
\end{aligned}
$$

An easy check shows $f_{x, y}$ is an isometry. If $T_{+}$and $T_{-}$are reversed, a similar definition will give an isometry.

For the second condition in the definition of a $\Lambda$-distance graph, we note that the condition can only fail if there exist $x, y \in T_{+}$and $z \in T_{-}$(or vice versa) with $\hat{d}(x, z)>0$ and $\hat{d}(y, z)>0$ such that $x$ and $z$ determine ends $e_{1}$ and $e_{2}$ and $y$ and $z$ determine ends $e_{1}$ and $e_{3}$ with $e_{2} \neq e_{3}$. However, by Proposition 2.1 this is impossible. Hence, $\Phi_{n}(\mathcal{T})$ is a $\Lambda$-distance graph.

Since $\Lambda$ is discrete, we can apply Proposition 4.3. It is immediate that $\Phi_{n}(\mathcal{T})$ has diameter $(1, n)$. By Proposition 2.1, we know that the girth of $\Phi_{n}(\mathcal{T})$ is at least $(2,2 n)$. Now examining a pair of apartments of $T_{+}$and $T_{-}$with the same ends, we see that this pair corresponds to a circuit of $\Phi_{n}(\mathcal{T})$ of length $(2,2 n)$. Hence the girth of $\Phi_{n}(\mathcal{T})$ is $(2,2 n)$. Lastly we need to check that $\Phi_{n}(\mathcal{T})$ has no circuits of length $(2,2 n+1)$. However, the existence of such a circuit would imply that there existed points $x \in T_{+}$and $y_{1}, y_{2} \in T_{-}$such that $\hat{d}\left(x, y_{i}\right)=0$ for $i=1,2$. This is a contradiction to condition 1 in the definition of a twin tree. Thus we have that $\Phi_{n}(\mathcal{T})$ is a $(1, n)$-gon.

Suppose conversely that $\mathcal{G}=(\mathcal{P}, d)$ is a generalized $(1, n)$-gon with $\Lambda=Z \times Z$ ordered lexicographically. Let $x, y \in \mathcal{P}$ be chosen so that $d(x, y)=(1, n)$. Define $\Psi_{x, y}(\mathcal{G})=\left(T_{+}, T_{-}, \hat{d}\right)$ as follows. Let

$$
T_{+}=\{a \in \mathcal{P} \mid d(a, x)=(0, m) \text { for some } m \in Z\},
$$

and let

$$
T_{-}=\{a \in \mathcal{P} \mid d(a, y)=(0, m) \text { for some } m \in Z\} .
$$

Let two elements $a, b \in T_{ \pm}$be adjacent if $d(a, b)=(0,1)$. It is immediate that $T_{+}$ and $T_{-}$are trees. Now for $a, b \in T_{+} \cup T_{-}$with $a$ and $b$ in different trees, define $\hat{d}(a, b)=k$, where $d(a, b)=(1, n-k)$. Then, $\Psi_{x, y}(\mathcal{G})$ is a twin tree.

We now have an understanding of what any $(1, n)$-gon looks like.

Theorem 4.6. Let $\Lambda=Z \times Z$, ordered lexicographically. For $\mathcal{G}=(\mathcal{P}, d) a(1, n)$ gon, define the graph $G=(V, E)$ with $V=\mathcal{P}$, and $E=\{(x, y) \mid d(x, y)=(0,1)\}$. Then $G$ is a forest of trees such that for any pair of sub-trees $T_{1}$ and $T_{2}$ of $G$, there is a codistance function defined as above from d twinning $T_{1}$ and $T_{2}$.

Proof. It is clear that $G$ is a forest since $\mathcal{G}$ has girth $(2,2 n)$. Now, let $T_{1}$ and $T_{2}$ be two trees in $G$. Then, $\mathcal{G}^{\prime}=\left(T_{1} \cup T_{2}, d\right)$ is also a generalized $(1, n)$-gon. To check this we need simply see that every minimal path connecting vertices $x$ and $y$ of $T_{1}$ and $T_{2}$ is contained in $T_{1} \cup T_{2}$. But this follows immediately, since if $z$ lies on such a minimal path, then $d(x, z)+d(y, z) \leq(1, n)$, implying that $z \in T_{1}$ or $z \in T_{2}$. 
Now, letting $x \in T_{1}$ and $y \in T_{2}$ be chosen such that $d(x, y)=(1, n)$, we then have that $\Psi_{x, y}(\mathcal{G})$ is a twin tree. Of course, $\Psi_{x, y}(\mathcal{G})=\left(T_{1}, T_{2}, \hat{d}\right)$, where $\hat{d}$ is defined as above.

Given a twin tree $\mathcal{T}$, we note that for any choices of $x, y \in \Phi_{n}(\mathcal{T})$ with $d_{n}(x, y)=$ $(1, k)$ for some $k \in Z$, we have $\Psi_{x, y}\left(\Phi_{n}(\mathcal{T})\right) \cong \mathcal{T}$. It may be the case, however, for a general $(1, n)$-gon that there are vertices $x, y, z \in \mathcal{P}$ such that $d(x, y), d(x, z)$ and $d(y, z)$ are all greater than $(0, m)$ for all $m \in Z$. Hence, there are $(1, n)$-gons corresponding to forests of more than two trees.

As a corollary of Theorem 4.5 and Theorem 3.2, we obtain with $\Lambda=Z \times Z$ ordered lexicographically:

Corollary 4.7. Given a twin tree $\mathcal{T}=\left(T_{+}, T_{-}, \hat{d}\right)$, there exist a $\Lambda$-tree $\mathcal{C}\left(\Phi_{n}(\mathcal{T})\right)$ "covering" $\mathcal{T}$, and a group $G$ of isometries of $\mathcal{C}\left(\Phi_{n}(\mathcal{T})\right)$ such that

$$
\Phi_{n}(\mathcal{T}) \cong \mathcal{C}\left(\Phi_{n}(\mathcal{T})\right) / G
$$

As a final note, we point out that the definition of a generalized $\lambda$-gon makes it easy to generalize the notion of a twin tree to $\Lambda$-trees. Given $\Lambda$ a totally ordered abelian group, let $\Lambda^{\prime}=Z \times \Lambda$ be ordered lexicographically. Let 0 also denote the zero element of $\Lambda$.

Definition. A twin $\Lambda$-tree is a generalized $(1,0)_{\Lambda^{\prime}}$-gon such that there exist points $x, y \in \mathcal{P}$ with $\min \{d(x, z), d(y, z)\}<(1, \lambda)$ for all $\lambda \in \Lambda$ and all $z \in \mathcal{P}$.

Applying a process like that used to define $\Psi_{x, y}(\mathcal{G})$ up above, a twin $\Lambda$-tree corresponds to a triple $\left(T_{x}, T_{y}, \hat{d}\right)$, where

$$
\begin{aligned}
& T_{x}=\{z \in \mathcal{P} \mid d(x, z)=(0, \lambda) \text { for some } \lambda \in \Lambda\}, \\
& T_{y}=\{z \in \mathcal{P} \mid d(y, z)=(0, \lambda) \text { for some } \lambda \in \Lambda\},
\end{aligned}
$$

and $\hat{d}: T_{x} \times T_{y} \cup T_{y} \times T_{x} \rightarrow \Lambda$ is defined so that $d(x, y)=(1,-\hat{d}(x, y))$.

Defining $\Phi_{0}$ for general $\Lambda$ as we did in the case $\Lambda=Z$ above, we have:

Definition. A twin $\Lambda$-tree is a triple $\mathcal{T}=\left(T_{+}, T_{-}, \hat{d}\right)$, where $T_{+}$and $T_{-}$are $\Lambda$ trees, and $\hat{d}: T_{x} \times T_{y} \cup T_{y} \times T_{x} \rightarrow \Lambda$ is a symmetric function such that $\Phi_{0}(\mathcal{T})$ is a generalized $(1,0)_{\Lambda^{\prime}}$-gon.

\section{Free Construction}

In this section we give a free construction of $\lambda_{\Lambda}$-gons when $\Lambda$ is discrete and satisfies one extra condition. Suppose $\Lambda$ is a discrete totally ordered abelian group with minimal element $1_{\Lambda}$. Suppose further that $\Lambda^{\prime} \subset \Lambda$ is a subgroup of index 2 not containing $1_{\Lambda}$. We note that if $\Lambda=\widetilde{\Lambda} \times\left\langle 1_{\Lambda}\right\rangle$, lexicographically ordered, then $\Lambda^{\prime}=\widetilde{\Lambda} \times\left\langle 2 \cdot 1_{\Lambda}\right\rangle$ is such a subgroup. We define an element $\alpha$ of $\Lambda$ to be even if $\alpha \in \Lambda^{\prime}$, and we define $\alpha$ to be odd if $\alpha \notin \Lambda^{\prime}$. Given $\alpha, \beta \in \Lambda$, it follows that the element $\alpha+\beta$ is even if and only if either both $\alpha$ and $\beta$ are even or both are odd. Choose $\lambda \in \Lambda$ with $\lambda>1_{\Lambda}$. For ease of notation, we will write $k$ in place of $k \cdot 1_{\Lambda}$.

In this case, we define a free construction based on the free construction of $n$-gons (see $[\mathrm{R}]$ for example). Begin with a $\Lambda$-graph $\mathcal{G}$ with diameter greater than $\lambda$ and no odd circuits. A $\Lambda$-graph $\mathcal{G}_{1}$ is created by connecting all pairs of vertices $(x, y)$ of distance greater than $\lambda$ by a path of length $\lambda-1$ if $d(x, y)+\lambda-1$ is even. The last condition implies that $\mathcal{G}_{1}$ has no odd circuits, and the first condition implies that if $x, y$ are vertices of $\mathcal{G}$, then $d_{\mathcal{G}_{1}}(x, y) \leq \lambda$. Inductively the $\Lambda$-graph $\mathcal{G}_{m+1}$ is created 
by the same process from the $\Lambda$-graph $\mathcal{G}_{m}$. It then will follow that $\bigcup_{m=1}^{\infty} \mathcal{G}_{m}$ is an $\lambda$-gon. In the case where $\Lambda=Z$, the argument is straightforward. Below we deal precisely with the more general case.

Let $\mathcal{G}=(\mathcal{P}, d)$ be a $\Lambda$-distance graph containing no circuits of odd length, and having diameter greater than $\lambda$. Suppose $a, b \in \mathcal{P}$ are such that $d(a, b) \geq \lambda+1$. We define $\mathcal{G}_{\lambda, a, b}=\left(\mathcal{P}^{\prime}, d^{\prime}\right)$, where

$$
\mathcal{P}^{\prime}=\mathcal{P} \cup\{(a, b, \alpha) \mid 0<\alpha<\lambda\}
$$

and

$$
d^{\prime}(x, y)= \begin{cases}\left|\alpha_{1}-\alpha_{2}\right|, & \left\{\begin{array}{l}
x=\left(a, b, \alpha_{1}\right), \\
y=\left(a, b, \alpha_{2}\right),
\end{array}\right. \\
\min \left\{\begin{array}{l}
d(x, a)+\alpha \\
d(x, b)+\lambda-\alpha
\end{array}\right\}, & \left\{\begin{array}{l}
x \in \mathcal{P}, \\
y=(a, b, \alpha),
\end{array}\right. \\
\min \left\{\begin{array}{l}
d(x, y) \\
d(x, a)+\lambda+d(y, b) \\
d(x, b)+\lambda+d(y, a)
\end{array}\right\}, & x, y \in \mathcal{P},\end{cases}
$$

extended symmetrically.

Proposition 5.1. $\mathcal{G}_{\lambda, a, b}$ is a $\Lambda$-graph.

Proof. A tedious but straightforward check shows that $d^{\prime}$ is a $\Lambda$-metric.

The isometry conditions remain. If $x, y \in \mathcal{P}$ and $d^{\prime}(x, y)=d(x, y)$, and $f \in \mathcal{F}_{\mathcal{G}}$ with $f(0)=x$ and $f\left(\lambda_{f}\right)=y$, then $f \in \mathcal{F}_{\mathcal{G}^{\prime}}$, as can be easily checked. If $d^{\prime}(x, y)=$ $d(x, a)+d(y, b)+\lambda$, define the isometry by

$$
\bar{f}_{x, y}(\alpha)= \begin{cases}f_{x, a}(\alpha), & \alpha \leq d(x, a) \\ (a, b, \alpha-d(x, a)), & d(x, a)<\alpha<d(x, a)+\lambda \\ f_{b, y}(\alpha-d(x, a)-\lambda), & d(x, a)+\lambda \leq \alpha \leq d^{\prime}(x, y)\end{cases}
$$

where $f_{u, v} \in \mathcal{F}_{\mathcal{G}}$ denotes the isometry from $u$ to $v$ in $\mathcal{G}$. To see that $\bar{f}_{x, y} \in \mathcal{F}_{\mathcal{G}^{\prime}}$, let $z, w \in\left[0, d^{\prime}(x, y)\right]$ with $z<w$. If $d^{\prime}(z, w)<|w-z|$, the triangle inequality would not hold for $d^{\prime}$. In each case for $z, w$, however, it is easy to verify that $d^{\prime}(z, w) \leq|w-z|$.

Next suppose $x \in \mathcal{P}$ and $y=(a, b, \beta)$. If $d^{\prime}(x, y)=d(x, a)+\alpha$, define

$$
\bar{f}_{x, y}(\alpha)= \begin{cases}f_{x, a}(\alpha), & \alpha \leq d(x, a), \\ (a, b, \alpha-d(x, a)), & d(x, a)<\alpha \leq d^{\prime}(x, y) .\end{cases}
$$

The same proof as before shows that $\bar{f}_{x, y} \in \mathcal{F}_{\mathcal{G}^{\prime}}$ is an isometry. Finally, if $x=$ $\left(a, b, \alpha_{1}\right)$ and $y=\left(a, b, \alpha_{2}\right)$ with $\alpha_{2}>\alpha_{1}$, define

$$
\bar{f}_{x, y}(\beta)=\left(a, b, \beta+\alpha_{1}\right)
$$

for $\beta \in\left[0, \alpha_{2}-\alpha_{1}\right]$. This is clearly an isometry. Since given an isometry $f$ with $f(0)=x$ and $f\left(\lambda_{f}\right)=y$, it follows that $f^{\prime}(\alpha)=f\left(\lambda_{f}-\alpha\right)$ is also an isometry, all the possible pairs $x, y$ are now covered.

It remains to check the Y-condition. Let $f, g \in \mathcal{F}_{\mathcal{G}_{\lambda, a, b}}$ be given. Note that any isometry $f \in \mathcal{F}_{\mathcal{G}_{\lambda, a, b}}$ can be broken into three (possibly trivial) pieces $f_{1}, f_{2}, f_{3}$ where $f_{1}, f_{3} \in \mathcal{F}_{\mathcal{G}}$ and $f_{2}$ is an isometry from an interval of $\Lambda$ to a subset of $\{(a, b, \alpha) \mid \alpha \in[0, \lambda-1]\}$ (where $(a, b, 0)=a$ and $(a, b, \lambda)=b)$. Suppose $f$ breaks into $f_{1}, f_{2}, f_{3}$ and $g$ into $g_{1}, g_{2}, g_{3}$. If $f\left(\lambda_{f}\right)=g(0)$, this implies that $f_{3}\left(\lambda_{f_{3}}\right)=g_{1}(0)$. If $a_{f_{3} g_{1}} \neq \lambda_{f_{3}}$ or $a_{f_{3} g_{1}} \neq \lambda_{g_{1}}$, then we let $a_{f g}=a_{f_{3} g_{1}}$, and we are done. If 
$a_{f_{3} g_{1}}=\lambda_{f_{3}}=\lambda_{g_{1}}$, then if $\lambda_{f_{2}} \neq \lambda_{g_{2}}$, we can choose $a_{f g}=\lambda_{g_{1}}+\min \left\{\lambda_{f_{2}}, \lambda_{g_{2}}\right\}$. Finally, if $a_{f_{3} g_{1}}=\lambda_{f_{3}}=\lambda_{g_{1}}$, and $\lambda=\lambda_{f_{2}}=\lambda_{g_{2}}$, we take $a_{f g}=a_{f_{1} g_{3}}+\lambda_{g_{1}}+\lambda$. In each of these cases, it is clear that $a_{f g}$ is an element of $\left[0, \lambda_{g}\right]$ satisfying the requirements of the $\mathrm{Y}$-condition, since the $\mathrm{Y}$-condition is satisfied in $\mathcal{G}$. Hence $\mathcal{G}_{\lambda, a, b}$ is a $\Lambda$-graph.

Since $\mathcal{G}_{\lambda, a, b} \cong \mathcal{G}_{\lambda, b, a}$, we define $\mathcal{G}_{\lambda,\{a, b\}}=\mathcal{G}_{\lambda, a, b}$. We now give a technical result that we will use later.

Proposition 5.2. Let $\mathcal{G}$ be a $\Lambda$-graph with no odd circuits, diameter greater than or equal to $\lambda+1$ and girth at least $2(\lambda+1)$. Suppose further $d(a, b)>\lambda+1$ and $d(a, b)+\lambda$ is even. Then $\mathcal{G}_{\lambda, a, b}$ is a $\Lambda$-graph with no odd circuits, diameter greater than or equal to $\lambda+1$ and girth at least $2(\lambda+1)$. Suppose $\{x, y\} \neq\{a, b\}$. Then

(5.2.1): if $d(x, y)>\lambda+1$, and $d(x, y)+\lambda$ is even, then $d^{\prime}(x, y)>\lambda+1$,

(5.2.2): if $d(x, y)>\lambda+1$, and $d(x, y)+\lambda$ is odd, then $d^{\prime}(x, y) \geq \lambda+1$, and

(5.2.3): if $d(x, y) \leq \lambda+1$, then $d^{\prime}(x, y)=d(x, y)$.

Proof. Suppose $x, y \in \mathcal{P}$ with $d(x, y)$ even. Since $\mathcal{G}$ has no odd circuits, $d(x, y)+$ $d(a, x)+d(b, y)+\lambda$ is even, and as a result, we see that $d^{\prime}(x, y)$ is even. Hence, since the parity of the distance of two points remains unchanged, $\mathcal{G}_{\lambda, a, b}$ has no odd circuits contained entirely in $\mathcal{P}$. If $d(x, y)>\lambda+1$, and $d(x, y)+\lambda$ is even, it follows that $d(x, a)+d(y, b)$ is even. If $\{x, y\} \neq\{a, b\}$, then $d(x, a)+d(y, b) \geq 2$, and hence,

$$
d^{\prime}(x, y)=\min \{d(x, y), d(x, a)+d(y, b)+\lambda, d(x, b)+d(y, a)+\lambda\} \geq \lambda+2,
$$

establishing (5.2.1). Results (5.2.2) and (5.2.3) follow immediately from the definition of $d^{\prime}$.

Suppose $C=x_{0}, x_{1}, \ldots, x_{n}=x_{0}$ is an odd circuit of $\mathcal{G}_{\lambda, a, b}$ containing a minimal number of elements not in $\mathcal{P}$. Without loss of generality, we may assume $x_{0}, x_{2} \in \mathcal{P}$ and $x_{1} \notin \mathcal{P}$. An easy check will verify that $d^{\prime}\left(x_{0}, x_{2}\right)+d^{\prime}\left(x_{0}, x_{1}\right)+d^{\prime}\left(x_{1}, x_{2}\right)$ is even. As a result, $C^{\prime}=x_{0}, x_{2}, x_{3}, \ldots, x_{n}$ is an odd circuit containing fewer members not in $\mathcal{P}$. Hence $\mathcal{G}_{\lambda, a, b}$ has no odd circuits. It is immediate that if $d(a, b)>\lambda+1$, then the diameter of $\mathcal{G}_{\lambda, a, b}$ is at least $\lambda+1$. It is also clear that the shortest circuit must still have length at least $2(\lambda+1)$.

Let

$$
S=\left\{\left\{a_{i}, b_{i}\right\} \in \mathcal{P} \times \mathcal{P} \mid d\left(a_{i}, b_{i}\right)>\lambda+1, i=1, \ldots, n\right\}
$$

be a finite set, and suppose $\{a, b\} \subset S$ implies $d(a, b)+\lambda$ is even. Define

$$
\mathcal{G}_{S, \lambda}=\left(\ldots\left(\left(\mathcal{G}_{\lambda, a_{1}, b_{1}}\right)_{\lambda, a_{2}, b_{2}}\right) \ldots\right)_{\lambda, a_{n}, b_{n}} .
$$

Proposition 5.3. If $\mathcal{G}$ is a $\Lambda$-graph with diameter at least $\lambda+1$ and no odd circuits, and if $S$ is as above, then $\mathcal{G}_{S, \lambda}$ also is a $\Lambda$-graph with no odd circuits having diameter at least $\lambda+1$. Moreover, $\mathcal{G}_{S, \lambda}$ is independent of the ordering of the elements of $S$.

Proof. Induction and the preceding propositions imply $\mathcal{G}_{S, \lambda}$ is a well-defined $\Lambda$ graph with no odd circuits. The final result can be verified by induction and an easy calculation on the distance function in the case $|S|=2$.

We are now ready to give the main step in the free construction of a $\lambda_{\Lambda}$-gon. Suppose $\mathcal{G}$ is a $\Lambda$-graph and having no odd circuits, diameter at least $\lambda+1$ and girth greater than $2 \lambda$. Define

$$
T(\mathcal{G})=\left\{\{a, b\} \subset \mathcal{P} \mid d_{\mathcal{G}}(a, b)>\lambda, \text { and } d_{\mathcal{G}}(a, b)+\lambda-1 \text { is even }\right\} .
$$


In general $T$ will not be finite. Define $\mathcal{G}_{T(\mathcal{G}), \lambda-1}=\left(\mathcal{P}_{T(\mathcal{G})}, d_{T(\mathcal{G})}\right)$ as follows: Let

$$
\begin{aligned}
\mathcal{P}_{T(\mathcal{G})} & =\bigcup_{\substack{J \subset T(\mathcal{G}) \\
|J|<\infty}} \mathcal{P}_{\mathcal{G}_{J, \lambda-1}}, \\
d_{T(\mathcal{G})}(x, y) & =\min _{\substack{x, y \in \mathcal{P}_{J} \\
|J|<\infty}}\left\{d_{\mathcal{G}_{J, \lambda-1}}(x, y)\right\} .
\end{aligned}
$$

Define $\mathcal{P}_{J}=\mathcal{P}_{\mathcal{G}_{J, \lambda-1}}$ and $d_{J}=d_{\mathcal{G}_{J, \lambda-1}}$ and $\mathcal{F}_{J}=\mathcal{F}_{\mathcal{G}_{J, \lambda-1}}$.

Proposition 5.4. With the above assumptions, $\mathcal{G}_{T(\mathcal{G}), \lambda-1}$ is a $\Lambda$-graph with diameter at least $\lambda$, girth at least $2 \lambda$ and having no odd circuits. Moreover, if $x, y \in \mathcal{P}$, then $d_{T(\mathcal{G})}(x, y) \leq \lambda$.

Proof. The idea of the proof is to reduce all definitions to $\mathcal{G}_{J, \lambda-1}$ for some finite subset $J$ of $T$. We first need to show that $d_{T(\mathcal{G})}(x, y)$ is well-defined for all pairs $(x, y) \in \mathcal{P}_{T(\mathcal{G})}$. Suppose $x, y \in \mathcal{P}$. If $d_{\mathcal{G}}(x, y) \leq \lambda$, then by Propisition $5.2 d_{J}(x, y)=$ $d_{\mathcal{G}}(x, y)$ for all finite subsets $J$ of $T$. If $d_{\mathcal{G}}(x, y)>\lambda$ and $d_{\mathcal{G}}(x, y)+\lambda-1$ is even, then for all finite subsets $J$ not containing $\{x, y\}$ Proposition 5.2 implies $d_{J}(x, y) \geq \lambda+1$. If $J$ contains $\{x, y\}$, however, then $d_{J}(x, y)=\lambda-1$ by the same proposition. Hence $d_{T(\mathcal{G})}(x, y)=\lambda-1$. If $d_{\mathcal{G}}(x, y)+\lambda-1$ is odd, Proposition 5.2 implies $d_{J}(x, y) \geq \lambda$ for all finite $J$. Hence it suffices to check that there exists a subset $J$ such that $d_{J}(x, y)=\lambda$. Let $z$ be such that $d_{\mathcal{G}}(y, z)=\lambda+1$ and $d_{\mathcal{G}}(x, z)=1$. Then, letting $J=\{y, z\}$, we have $d_{J}(x, y)=\lambda$.

If $x$ or $y$ is not in $\mathcal{P}$, let $K$ be minimal such that $x, y \in \mathcal{P}_{K}(|K| \leq 2)$. We will check the special case where $x=(a, b, \alpha)$ and $y=(c, d, \beta)$. In this case, if $K \subset J$, we have

$$
d_{K}(x, y)=\min \left\{\begin{array}{l}
d_{K}(a, c)+\alpha+\beta \\
d_{K}(a, d)+\alpha+(\lambda-1-\beta), \\
d_{K}(b, c)+(\lambda-1-\alpha)+\beta \\
d_{K}(b, d)+(\lambda-1-\alpha)+(\lambda-1-\beta)
\end{array}\right\}
$$

Since each term has a minimum over finite $K$, collectively there is a minimum. Hence $d_{K}(x, y)$ is well-defined. The case where $x \in \mathcal{P}$ and $y \notin \mathcal{P}$ works similarly. Hence, $d_{T(\mathcal{G})}$ is well defined. Now if $x, y \in \mathcal{P}$, and $d(x, y)>\lambda$, then either $d(x, y)+$ $\lambda-1$ is even, in which case $d_{T(\mathcal{G})}(x, y)=\lambda-1$, or there is an $x^{\prime}$ with $d\left(x^{\prime}, y\right)+\lambda-1$ even and $d\left(x^{\prime}, y\right)>\lambda$, implying $d_{T(\mathcal{G})}(x, y)=\lambda$, implying the last conclusion of the theorem. An easy check then shows that the diameter of $\mathcal{G}_{T(\mathcal{G})}$ is less than $2 \lambda$.

For the remainder of the proof we will write $d_{T}$ for $d_{T(\mathcal{G})}$.

By definition $d_{T}(x, x)=0$ and $d_{T}(x, y)=d_{T}(y, x)$. To see that $d_{T}$ is a $\Lambda$-metric it remains to show the triangle inequality. Let $x, y, z \in \mathcal{P}_{T}$. Then there exists a finite subset $J$ of $T$ such that $d_{T}=d_{J}$ on pairs from the set $\{x, y, z\}$. Since the triangle inequality holds for $d_{J}$, by Proposition 5.2, the triangle inequality holds for $d_{T}$ on these three. Hence $d_{T}$ is a $\Lambda$-metric.

We now show the two isometry conditions. For the existence of an isometry from $\left[0, d_{T}(x, y)\right]$ to $\mathcal{G}_{T(\mathcal{G})}$, we let $J$ be a finite subset of $T$ such that $d_{J}(x, y)$ is minimal. Let $f \in F_{J}$ be the isometry in $\mathcal{G}_{J}$ from $x$ to $y$, and let $a, b$ be in the image of $f$ with $f^{-1}(a)<f^{-1}(b)$. If $d_{T}(a, b)<d_{J}(a, b)$, then we would have $d_{T}(x, a)+d_{T}(a, b)+d_{T}(b, y)<d_{T}(x, y)$, violating the triangle inequality. Hence $d_{T}(a, b) \geq d_{J}(a, b)$, implying $d_{T}(a, b)=d_{J}(a, b)$, and $f \in \mathcal{F}_{\mathcal{G}_{T(\mathcal{G})}}$.

Let $f, g \in \mathcal{F}_{\mathcal{G}_{T(\mathcal{G})}}$ be isometries with $f\left(\lambda_{f}\right)=g(0)$. If we can show there exists a finite $J$ such that $f, g \in \mathcal{F}_{J}$, the Y-condition would follow from Proposition 5.3. 
If $f$ is an isometry for $\mathcal{G}_{T(\mathcal{G})}$ whose image contains one point of the form $(a, b, \alpha)$, either $f$ contains every point of that form, or $f$ begins or stops at such a point and $\lambda_{f} \leq 2 \lambda$. Hence the image of $f$ must be contained in $G_{J_{f}}$ for some subset $J_{f}$ of $T$ with at most three elements. Our goal is to show that $f$ is an isometry for $\mathcal{G}_{J_{f}}$. To prove this, we will need the following lemma:

Lemma 5.5. Suppose $f:[0, \gamma] \rightarrow \mathcal{G}_{T(\mathcal{G})}$ is an isometry with $f(0)=(a, b, \alpha)=y \notin$ $\mathcal{P}$ and $f(\gamma) \in \mathcal{P}$. If $f(\eta)=(a, b, \beta)=x$, then $a \in \operatorname{Im}(f)$ if $\beta<\alpha$ and $b \in \operatorname{Im}(f)$ if $\beta>\alpha$.

Proof. Suppose $\beta<\alpha$ and consider $z=f^{-1}(\alpha)$. Then, $d_{T}(z, y)=\alpha$, and $d_{T}(z, x)=\beta=\alpha-\eta$. Moreover, there exists a finite subset $I$ of $T$ such that $d_{I}(x, z)=d_{T}(x, z)$ and $d_{I}(x, y)=d_{T}(x, y)$. But we must have $z \in \mathcal{P}_{I-\{a, b\}}$, and hence $d_{I}(x, z)=\min \left\{d_{I-\{a, b\}}(z, a)+\alpha, d_{I-\{a, b\}}(z, b)+\lambda-1-\alpha\right\}$. The condition $\beta<\alpha$ implies that the the minimum is achieved in the first case, and hence $d_{I-\{a, b\}}(a, z)=0$, implying $a=z$. The other case is similar.

Suppose $d_{J}(x, y)>d_{T}(x, y)$ and $x, y \in \operatorname{Im}(f)$. It follows from Proposition 5.2 that $d_{J}(x, y)>\lambda$. Moreover, by the lemma, we may assume that $x, y \in \mathcal{P}$ (replacing $(a, b, \alpha)$ by $a$ or $b$ as appropriate). If $d_{T}(x, y)<\lambda-1$, then $d_{J}(x, y)=d(x, y)<\lambda-1$, and we may assume $d_{T}(x, y)=\lambda-1$ or $\lambda$. In the first case, Proposition 5.2 implies that the image of $f$ contains a point of the form $(x, y, \alpha)$ or $(y, x, \alpha)$, and hence $\{x, y\} \in J$ and $d_{J}(x, y)=\lambda-1$. If $d_{T}(x, y)=\lambda$, let $z=f(1)$. If $z \in \mathcal{P}$, then $d_{T}(z, y)=\lambda-1$, implying by the previous sentence that $d_{J}(z, y)=\lambda-1$ and hence $d_{J}(x, y)=\lambda$. The other possibility is that $z=(x, w, 1)$. In this case, it follows that $d(y, w)=1$ and that $\{x, w\} \subset J$. Hence $d_{J}(x, y)=d_{T}(x, y)$, and $f$ is an isometry for $\mathcal{G}_{J_{f}, \lambda-1}$. Let $I=J_{f} \cup J_{g}$. Then, $f$ and $g$ are both isometries for $\mathcal{G}_{I, \lambda-1}$. Since $\mathcal{G}_{I, \lambda-1}$ is a $\Lambda$-graph, there exists $a_{f g}$ satisfying the Y-condition.

Let $\mathcal{G}_{0}$ be any $\Lambda$-graph with no odd circuits, having diameter greater than $\lambda$, and girth greater than or equal to $2 \lambda$. Define $\mathcal{G}_{1}=\mathcal{G}_{T\left(\mathcal{G}_{0}\right), \lambda-1}$ and inductively define $\mathcal{G}_{i}=\mathcal{G}_{T\left(\mathcal{G}_{i-1}\right), \lambda-1}$. Write $\mathcal{G}_{i}=\left(\mathcal{P}_{i}, d_{i}\right)$ and $\mathcal{F}_{i}=\mathcal{F}_{\mathcal{G}_{i}}$.

Theorem 5.6. With the above definitions, let $H$ be the $\Lambda$-graph with $\mathcal{P}(H)=$ $\bigcup_{i=1}^{\infty} \mathcal{P}_{i}$ and $d_{H}(x, y)=\min _{i}\left\{d_{i}(x, y)\right\}$. Then $H$ is a $\lambda_{\Lambda}$-gon.

Proof. To see that $d_{H}$ is a well-defined $\Lambda$-metric, we note that Proposition 5.4 implies that if $x, y \in \mathcal{P}_{i}$, then $d_{i+1}(x, y) \leq \lambda$. It follows from Proposition 5.2 that $d_{j}(x, y)=d_{i+1}(x, y)$ for all $j>i$, and hence that the minimum exists. For the triangle inequality, given $x, y, z \in \mathcal{P}(H)$ there exists $i \in Z$ such that $x, y, z \in \mathcal{P}_{i}$ and such that $\left.d_{H}\right|_{\{x, y, z\}}=\left.d_{i}\right|_{\{x, y, z\}}$. Hence $d_{H}(x, y)+d_{H}(y, z) \geq d_{H}(x, z)$ follows from the triangle inequality for $d_{i}$. It is now clear that the diameter of $H$ is $\lambda$.

To check the isometry conditions we apply the same technique as in the proof of Proposition 5.4. Given $x, y \in \mathcal{P}(H)$, then there exists $i$ such that $d_{i}(x, y)=$ $d_{H}(x, y)$. Since $\mathcal{G}_{i}$ is a $\Lambda$-graph, there exists $f \in \mathcal{F}_{i}$ with $f(0)=x$ and $f\left(d_{i}(x, y)\right)=$ $y$. If $a, b \in \operatorname{Image}(f)$ then $d_{i}(a, b) \leq \lambda$. This implies by Proposition 5.2 that $d_{i}(a, b)=d_{H}(a, b)$ and, as a result, $f \in \mathcal{F}_{H}$. For the Y-condition, we need to check that if $f \in \mathcal{F}_{H}$, then $f \in \mathcal{F}_{i}$ for some $i$. Let $x=f(0)$ and $y=f\left(\lambda_{f}\right)$. Since the diameter of $H$ is $\lambda$, we know $d_{H}(x, y)=\lambda_{f} \leq \lambda$. Let $z=f(1)$. Then $d_{H}(z, y) \leq \lambda-1$. Let $i$ be such that $z, y \in \mathcal{P}_{i}$ and $d_{i}(z, y)=d_{H}(z, y)$, and let $j \geq i$ be such that $d_{j}(x, y)=d_{H}(x, y)$. Choose $g \in \mathcal{G}_{j}$ such that $g(0)=x, g(1)=z$, and $g\left(d_{i}(x, y)\right)=y$. We will show that $f=g$. Let $a=f(\beta)$, and let $k \geq j$ be such that 
$d_{k}(a, y)=d_{H}(a, y)$ and $d_{k}(a, z)=d_{H}(a, z)$. If $f(\beta) \neq g(\beta)$ then $f$ and $g$ determine a circuit of $\mathcal{G}_{k}$ of length less than $2 \lambda$. This contradicts Proposition 5.4. Hence $f(\beta)=g(\beta)$ for all $\beta \in\left[0, \lambda_{f}\right]$, and $f=g \in \mathcal{F}_{\mathcal{G}_{j}}$. Given two isometries $f, g \in \mathcal{F}_{H}$, there exists an $i$ such that $f, g \in \mathcal{F}_{i}$. Since $\mathcal{G}_{i}$ is a $\Lambda$-graph, we have there exists $a_{f g}$ satisfying the Y-condition.

It remains to show that $H$ has girth $2 \lambda$ and no odd circuits. By the hypothesis, there exists a circuit of length $2 \lambda$ in $\mathcal{G}_{1}$ and hence in $H$. Any circuit can consist of the non-backtracking concatenation of a finite number of elements of $\mathcal{F}_{H}$. Hence, by the above argument, any circuit is also a circuit of $\mathcal{G}_{i}$ for large enough $i$. As a result, any circuit of $H$ must be both even and have length greater than or equal to $2 \lambda$. Hence $H$ is a $\lambda_{\Lambda}$-gon.

While this proof takes any $\Lambda$-graph of sufficient girth and diameter and embeds it in a $\lambda_{\Lambda}$-gon, it does have one drawback. In all cases, the $\lambda_{\Lambda}$-gon produced has infinite valence. That is, given $x \in \mathcal{P}$, the set $\{y \mid d(x, y)=1\}$ is infinite.

\section{Further Questions}

We conclude by listing some open questions concerning $\lambda$-gons.

Question 1. Modify the above free construction so that it will work for more general $\Lambda$. In particular, we would like to generalize this construction to the case where $\Lambda$ is not discrete.

Such a free construction would provide some non-trivial examples of $\lambda_{\Lambda}$-gons beyond the basic ones. We note that in [Be], there is a free construction given for a $1_{Q}$-gon (properly interpreted). It is probably possible to generalize this construction to the case where the smallest convex subgroup of $\Lambda$ is isomorphic to the rationals. However, I do not see how it can be done otherwise.

Question 2. Find a $(1,0)_{Z \times Z}$-gon with $\mathrm{SL}_{2}\left(Q_{p}\right)$ acting on it naturally.

This question is not really well-posed, as we don't say what we mean by a "natural action" in this case. The ideas we have at present would require a slightly more general construction than a $Z \times Z$-gon.

Question 3. Find examples arising from groups that do not come from twin trees.

Here is a wide open question. It seems reasonable that such examples exist, but at the present time I am somewhat stymied by this question.

Question 4. Give a free construction producing $\lambda_{\Lambda}$-gons of finite valence.

\section{REFERENCES}

[AB] R. Alperin and H. Bass, Length Functions of Group Actions on $\Lambda$-trees, Proceedings of the 1984 Alta Conference on Combinatorial Group Theory and Topology, Annals of Mathematical Studies, vol. 111, Princeton Univ. Press, Princeton, NJ, 1987, pp. 265-378. MR 89c:20057

[B] S.A. Basarab, On a Problem Raised by Alperin and Bass, in Arboreal Group Theory, (ed. R. Alperin), Mathematical Sciences Research Institute Publications 19, Springer-Verlag, New York, 1991, pp. 35-68. MR 92b:57001

[Be] C. Bennett, Generalized Spherical Buildings, preprint.

[K] W. Kantor, Generalized Polygons, SCABs and GABs, in Buildings and the Geometry of Diagrams (ed. L.A. Rosati), Lecture Notes in Mathematics 1181, Springer-Verlag, Berlin, 1986, pp. 79-158. MR 87k:51014

[MS] J. Morgan and P. Shalen, Valuations, Trees, and Degenerations of Hyperbolic Structures, I, Annals of Mathematics 120 (1984), 401-476. MR 86f:57011 
[R] M. Ronan, Lectures on Buildins, Academic Press, San Diego (1989). MR 80j:20001

[RT] M. Ronan and J. Tits, Twin Trees, I, Inventiones Mathematicae 116 (1994), 463-479. MR 94k:20058.

[S] J-P. Serre, Trees, Springer-Verlag, Berlin, 1980. MR 82c:20083

[T1] J. Tits, Buildings of Spherical Type and Finite BN-Pairs, Lecture Notes in Mathematics, 386, Springer-Verlag, Berlin (1974). MR 57:9866

[T2] J. Tits, Course Notes on Twin Buildings Annuaire du Collège de France, 89e année, 19881989, 81-95.

[T3] J. Tits, Course Notes on Twin Buildings Annuaire du Collège de France, 90e année, 19891990, 87-103.

[T4] J. Tits, Twin Buildings and Groups of Kac-Moody Type, in Groups, Combinatorics, and Geometry, Durham 1990 (ed. M. Liebeck and J. Saxl), London Mathematical Society Lecture Notes 165, Cambridge University Press, 1992, pp. 249-286. MR 94d:20030

Department of Mathematics and Statistics, Bowling Green State University, BowlING Green, Ohio 43403

E-mail address: cbennet@andy.bgsu.edu 